\title{
Channel Model for the Surface Ducts: Large-scale Path-Loss, Delay Spread, and AOA
}

\author{
Ergin Dinc, Student Member, IEEE, Ozgur B. Akan, Senior Member, IEEE
}

\begin{abstract}
Atmospheric ducts, that are caused by the rapid decrease in the refractive index of the lower atmosphere, can trap the propagating signals. The trapping effects of the atmospheric ducts can be utilized as a communication medium for beyondLine-of-Sight (b-LoS) links. Although the wave propagation and the refractivity estimation techniques for the atmospheric ducts are well studied, there is no work which provides a channel model for the atmospheric ducts. Therefore, we develop a large-scale path-loss model for the surface ducts based on the parabolic equation (PE) methods for the first time in the literature. In addition, we develop a ray-optics (RO) method to analyze the delay spread and angle-of-arrival (AOA) of the ducting channel with the surface ducts. By using the developed RO method, we derive an analytical expression for the effective trapping beamwidth of the transmitter to predict the ranges of the beamwidth that can be trapped by the surface ducts according to the refractivity and the channel parameters.
\end{abstract}

Index Terms-Propagation, Refraction, Communication channels, Ray tracing, Delay estimation, Surface duct

\section{INTRODUCTION}

A TMOSPHERIC ducts are caused by non-standard atmospheric conditions, and wave propagation in the presence of atmospheric ducts is called as anomalous wave propagation [1]. An atmospheric duct is a layer in which rapid decrease in the refractivity of the lower atmosphere occur. In this way, atmospheric ducts can trap the propagating signals as shown in Figure 1. Most of the signal energy propagates in ducting layer unlike standard atmosphere. Therefore, trapped signals can propagate through beyond-Line-of-Sight (b-LoS) distances with lower path-loss values.

Modern naval b-LoS systems mostly utilize Satellite Communications (SATCOM). However, SATCOM has capacity problems under low coverage and high transmission delays. In addition, the range of direct communication systems depends on the height of transmitter towers. Generally, it is expensive or not possible to use high transmitter towers to monitor coastal areas. Therefore, [2] provides the implementation of ducting channel for a $78 \mathrm{~km}$ link with low height antennas to monitor a reef site. According to their results, atmospheric ducts can provide $10 \mathrm{Mbps}$ for $80 \%$ of the time at $10.5 \mathrm{GHz}$. Based on our reviews [3], ducting channel b-LoS communications can connect distances up to $500-1000 \mathrm{~km}$ in coastal and maritime environments because duct formation is more probable in regions with high humidity.

E. Dinc and O. B. Akan are with the Next-generation and Wireless Communications Laboratory, Department of Electrical and Electronics Engineering, Koc University, Istanbul, 34450 Turkey (e-mail: edinc@ku.edu.tr; akan@ku.edu.tr).
Although wave propagation and refractivity modeling of atmospheric ducts are well-studied topics [3], there is no work which considers channel modeling for atmospheric ducts. In addition, available studies cannot estimate large-scale and small-scale characteristics of ducting channels. Since ducting channel communication can be a promising candidate for $b-$ LoS communications, we aim to develop a large-scale pathloss model for surface ducts and analyze delay spread and AOA of surface ducts for the first time in the literature. In this way, development of large-scale path-loss model enables us to make link budget calculations faster for different channel and duct parameters. In addition, small-scale path-loss, fading, characteristics of the channel can be predicted as well.

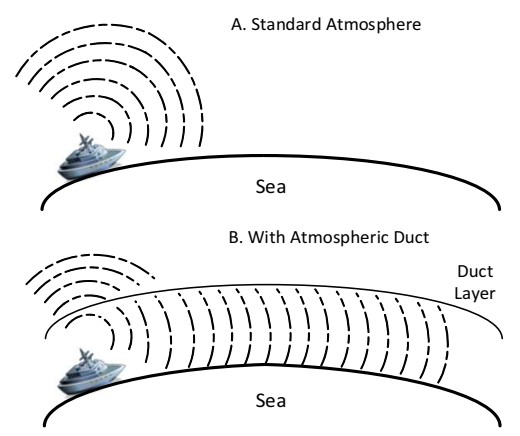

Fig. 1. Signal spreading in the standard atmosphere and the duct.

The contributions of this paper is tri-fold. Firstly, we develop a statistical large-scale path-loss model for surface ducts based on parabolic equation (PE) simulations. The developed large-scale path-loss model is utilized to predict the distribution of small-scale fading, and communication ranges that can be provided by surface ducts via link budget analysis. We utilize PE methods to estimate the path-loss for atmospheric ducts because ray-tracing and normal modes techniques are both unreliable and too resource consuming under non-standard atmospheric conditions [4]. The proposed large-scale pathloss model can estimate path-loss results for changing duct heights and channel parameters. Therefore, the developed method can provide fast estimates of path-loss under varying channel conditions. In addition, we develop a ray-optics (RO) method to calculate ray trajectories with surface ducts. By using the developed method, we analyze delay spreads to determine the fading behavior of the channel. Available studies do not provide a realistic analysis for the delay spread of ducting channels. Lastly, we develop an analytical formula to determine the effective trapping beam-width, which represents the range of beam-widths that can be trapped by surface 
ducts according to the channel conditions. Signals cannot be trapped by surface ducts beyond the effective trapping beam-width, and their power propagates through troposphere. Energy of these signals is wasted in b-LoS systems. For this reason, we develop an analytical formula which can help both academia and system designers while designing efficient bLoS communication links with atmospheric ducts.

The remainder of the paper is organized as follows. Section II provides related works. Section III includes refractivity models of atmospheric ducts. In Section IV, we review both PE methods and RO methods to model surface ducts. In addition, this section includes the derivation of RO methods for surface ducts and the effective trapping beam-width. Section $\mathrm{V}$ provides the derivation of large-scale path-loss model for surface ducts. In Section VI, simulation results are presented for delay spread, AOA and path-loss of the channel under various cases. Lastly, conclusions are presented in Section VII.

\section{RELATED WORK}

Ducting channel studies can be divided into two main parts: refractivity estimation and wave propagation studies. Refractivity estimation techniques aim to predict the vertical refractivity gradient which is essential to model the wave propagation at the lower atmosphere. There are various methods for predicting the refractivity of the lower atmosphere: the direct measurement techniques like microwave refractometers and radiosonde balloons [5], LIDAR (Light Detection and Ranging) techniques [6], in situ bulk measurements and meteorological methods [7], GPS (Global Positioning System) measurements [8] and refractivity from clutter techniques [9], [10]. In this study, we utilize the refractivity measurement results presented in [11] which is based on the atmospheric measurements in Istanbul, Turkey and, [12] which is based on the microwave refractometer measurements with a helicopter in Wallops Island, Virginia, USA. We determine the parameters regarding to characterize the surface ducts as suggested in [11], [12].

Since RO based techniques [13] or normal-modes based techniques [13], [14] are unreliable or too resource consuming, ducting channel propagation studies mostly focus on PE methods which utilize the paraxial approximation to the wave equation. PE can be solved with numerical methods: Split-Step Fourier (SSF), Finite Difference (FD) and Finite Element (FE). Especially with the development of SSF methods [15], PE has become the most popular method to model anomalous wave propagation [1], [2], [16], [17] because SSF methods provide fast and reliable results. In addition, PE methods can take the refractivity conditions as input and they can effectively model the complex boundary conditions. Good review of ducting channel wave propagation modeling and SSF based PE method can be found in [3] and [4], respectively.

To model wave propagation in ducting channel, there are available wave propagation tools. The most widely used one is AREPS that is developed by the Atmospheric Propagation Branch at the Space and Naval Warfare Systems Center, San Diego [18]. Combination of RO and PE methods is also promising as suggested in [19]-[22]. Therefore, AREPS utilizes both RO and SSF based PE methods. In addition,
PETOOL [23], which is also based on SSF, is capable of modeling both forward and backward scattered waves under different terrain and refractivity conditions. Therefore, PETOOL is promising especially for regions with irregular terrain conditions. Since PETOOL is a free and on-line available tool and it is calibrated with RO methods and AREPS as described in [23], we utilize PETOOL to model the surface ducts. In addition, we make RO analysis to the surface ducts to estimate the delay and AOA of the channel. Therefore, characteristics of the channel can be estimated with hybrid methods as suggested in [19], [20].

There are experimental studies about ducting channel wave propagation. [2] provides a high data rate employment of the atmospheric ducts for b-LoS sensor networks. In [2], the authors observed that wave propagation analysis performed in AREPS has strong consistency with the experimental results. In addition, [24]-[27] compare the simulation results with the PE methods and the experimental measurements for an $83 \mathrm{~km}$ link at 4.7,10 and $15 \mathrm{GHz}$. According to their results, the region had strong evaporation ducts, and the experimental pathloss measurements were consistent with the PE simulations. Furthermore, [1] provides one of the most extensive review of ducting channel with comparison of PE based results and experimental results for surface-based ducts. The results of this work show that surface-based ducts can provide 10-20 dB less path-loss compared to free space at b-LoS distances. Similar experimental results to verify PE simulations is presented in [28] as well. To sum up, the results of PE methods are validated through a number of different experimental works for different type of ducts. As a result, theoretical results that are generated with PE based tools can be considered as reliable based on previous experimental validations.

\section{REFRACTIVITY}

Atmospheric ducts are formed by non-standard atmospheric conditions. Formation and characteristic of atmospheric ducts depend on refractivity of the lower atmosphere. Therefore, we review refractivity and modified refractivity to characterize atmospheric ducts.

Tropospheric radio refractive index $(n)$ changes slightly with the atmospheric parameters: wind, temperature, pressure and humidity at the lower atmosphere. $n$ varies in the order of $10^{-3}$. Therefore, refractivity is utilized instead of refractive index and refractivity $(N)$ is given as [29]

$$
N=(n-1) \times 10^{6} \mathrm{~N} \text {-units, }
$$

where $n$ is the atmospheric refractive index.

Trapped signals in ducting layer has low grazing angles. Therefore, refractivity model should consider the curvature of the earth. Thus, modified refractivity $(M)$ is defined as [29]

$$
\begin{aligned}
M & =N+\left(\frac{h}{R_{0}}\right) \times 10^{6}, \\
& =N+157 \times h \text { M-units, }
\end{aligned}
$$

where $h$ is the height above the surface level in $\mathrm{km}$ and $R_{0}$ is the earth radius in $\mathrm{km}$. 
According to modified refractivity gradient, there four different refractive conditions. In sub-refraction condition $(\partial M / \partial z>157 \mathrm{M}$-units/km), signal rays are refracted away from ground through atmosphere. Standard refractivity condition is associated with (157 M-units/km $\geq \partial M / \partial z>$ $78 \mathrm{M}$-units $/ \mathrm{km}$ ) and this condition represents standard wave propagation. Signals propagate downward in super-refraction condition (78 M-units $/ \mathrm{km} \geq \partial M / \partial z>0$ M-units $/ \mathrm{km}$ ). Most importantly, trapping condition, which is also known as ducting condition, is associated with $0 \geq \partial M / \partial z$, and signal rays are trapped between surface and ducting layer. This effect can make trapped signals to propagate over-the-horizon with low path-loss. For the formation of atmospheric ducts, humidity is an essential factor [3]. Thus, ducting channel is a promising candidate for b-LoS communications especially in coastal and maritime environments as reviewed in [3].

There are four type of atmospheric ducts according to their formation process and refractivity profiles: evaporation ducts, surface-based ducts, elevated ducts, and surface ducts. As in Figure 2(a), the modified refractivity of evaporation ducts can be modeled with a logarithmic function [30]

$$
M(z)=M_{0}+0.125 z-0.125 \delta \ln \left(\frac{z+z_{0}}{z_{0}}\right),
$$

where $M_{0}$ is the value of modified refractivity at the surface which is taken as 315 M-units [31], $z$ is the vertical height, $\delta$ is the duct height and $z_{0}$ is the aerodynamic roughness length which is assumed as $1.5 \times 10^{-4} \mathrm{~m}$ [30].

Surface-based and elevated ducts are modeled with a trilinear curve as in Figure 2. Surface ducts are modeled with a bi-linear curve as in Figure 2(c). Since evaporation ducts are modeled with the logarithmic function, evaporation ducts are complex to be analyzed with RO based tools. As a result, we only consider surface ducts in this paper. However, an evaporation duct and a surface duct having the same duct height is expected to have similar maximum delay spread and AOA because their refractivity profiles are closer as in Figure 2. Thus, results presented for surface ducts can be utilized to predict the characteristics of evaporation ducts as well.

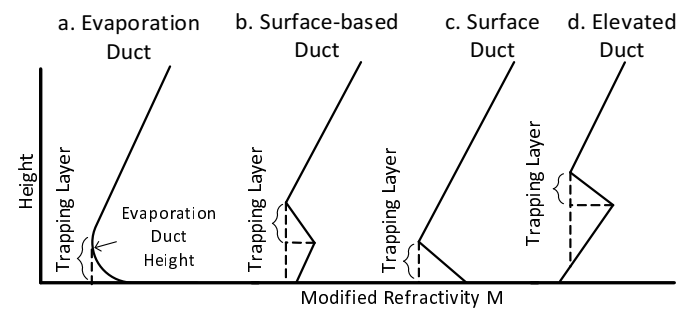

Fig. 2. Modified refractivity profiles of atmospheric ducts.

In this paper, surface ducts are characterize with two atmospheric parameters: duct height $(\delta)$ and duct strength $(\Delta M)$. Duct height is the height where the gradient of modified refractivity changes its sign as in Figure 2(c). Duct strength is the amount of change in modified refractivity from the bottom to the top of the surface duct. Modified refractivity above surface duct is assumed as standard condition and decreases with $118 \mathrm{M}$-units $/ \mathrm{km}$. We utilize the experimental surface duct measurements [11], [12] to determine duct height and duct strength parameters.

\section{Channel Modeling Techniques for Surface DUCTS}

Ducting channel wave propagation requires modeling of atmospheric parameters and complex boundary problems. Therefore, PE methods are preferred for estimating path-loss in ducting channels. In addition, we utilize RO methods to analyze delay spread and AOA of ducting channel with surface ducts. In this section, we first review PE, and develop a RO based method to analyze delay spread and AOA of ducting channels.

\section{A. Parabolic Equation (PE) Methods}

PE methods use paraxial approximation to the Helmholtz wave equation, and it was originally developed in [32]. PE methods are capable of modeling the complex boundary conditions and the refractivity variations of the lower atmosphere [33]. Especially with the derivation of a simple solution to PE based on Split-Step Fourier (SSF) method [15], PE methods became the dominant technique to model the wave propagation in ducting channels. Although there are other numerical methods to solve PE: finite difference (FD) and finite element (FE), SSF method is more preferable with its accuracy and computational efficiency [1], [3], [4], [18], [34].

Formulation of $\mathrm{PE}$ for the electromagnetic problems is summarized in [35]. The theory of PE is explained in detail in [1], [33], [36]. Interested reader may refer to these references. However, we do not discuss the theory of PE in this paper. Instead, we review how path-loss calculations are performed with PE methods. For 2D narrow angle forward scatter waves, $\mathrm{PE}$ in scalar form is given as [4]

$$
\frac{\partial u(x, z)}{\partial x}=\frac{i}{2 k} \frac{\partial^{2} u(x, z)}{\partial z^{2}}+\frac{i k}{2}\left(m^{2}(x, z)-1\right) u(x, z),
$$

where $k$ is the wave number, $m=1+M 10^{-6}$ is the modified refractive index, $u(x, z)$ is the reduced function, $x$ represents the horizontal axis, and $z$ represents the vertical axis (height). (4) can be used for both horizontal and vertical polarization.

We utilize PETOOL [23], which is a free on-line available tool based on the PE method with SSF, to solve (4). As discussed in Section II, PETOOL is calibrated with AREPS which is an experimentally validated tool. With PE methods, path-loss $(P L)$ can be found by using $u(x, z)$ as [33]

$$
P L=20 \log (4 \pi /|u(x, z)|)+10 \log (r)-30 \log (\lambda),
$$

where, $\lambda$ is the wavelength, and $r$ is the range. Unlike available propagation studies, we utilize PE methods to develop a largescale path-loss model for the first time in the literature in Section V.

\section{B. Ray-optics (RO) Methods}

Although PE methods dominate ducting channel wave propagation modeling, RO methods can be utilized to estimate delay spreads and propagation angles [33], [37] as in hybrid 


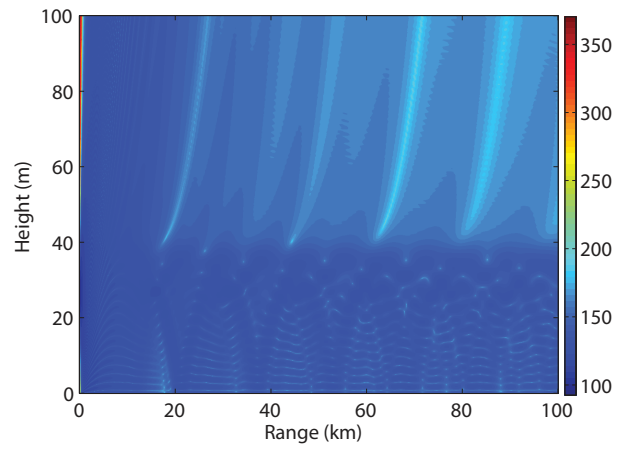

(a)

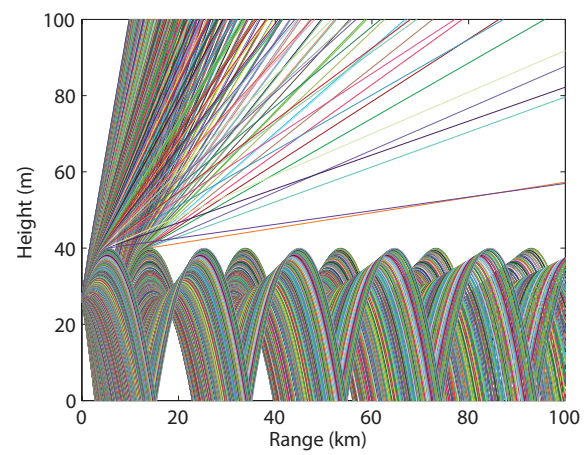

(b)

Fig. 3. Wave propagation results with (a) PETOOL, (b) RO methods for $40 \mathrm{~m}$ surface duct, $10 \mathrm{Ghz}, 27 \mathrm{~m}$ transmitter height.

models [18], [19]. In addition, [38] provides the comparison of AOA predictions with RO methods and the experimental observations. According to their results, the predicted AOA values were consistent with the observations. Since PE methods are not capable of estimating delay spreads and AOAs, RO methods are promising for the channel modeling in ducting layer.

RO methods utilize the well-known Snell's equation. By solving the Snell's equation for stratified atmosphere, the Eikonal equation is derived to calculate trajectory of each ray. Trajectories of rays are utilized to calculate delay spreads and AOA of the channel. There are some studies in the literature which focus on ray-tracing simulations to model wave propagation in atmospheric ducts. In [39], [40], raytracing analysis are performed to estimate delay in elevated ducts, but their models assume that only three rays can reach to the receiver. According to these papers, an $80 \mathrm{~km} \mathrm{link}$ with elevated ducts shows a few nanosecond delay spread. In [41], [42], they assume that odd number of rays can reach to the receiver and estimate AOA with the ray-tracing simulations for surface-based ducts. Although existing studies have significant results for ducting channel, more realistic results can be generated with RO methods. Therefore, we utilize RO methods to estimate the fading characteristics of the channel and the distribution of AOA for surface ducts. Lastly and more importantly, we derive an analytical expression for the maximum and minimum value of the angle-of-departure (AOD) which can be trapped by surface ducts. With the derived expression, transmitter can be designed to maximize the efficiency of the system by only propagate in the calculated beam-width span.

The remaining of the subsection includes derivation of ray trajectory formula and derivation of trapping beam-width:

1) Ray Trajectories: Ray trajectories in atmospheric ducts can be estimated with RO methods [37]. However, RO methods become more reliable above $3 \mathrm{GHz}$ [29]. Since evaporation ducts perform with lowest path-loss near $10 \mathrm{GHz}$ [2], [3], [16], we aim to focus $5-15 \mathrm{GHz}$ frequency range in this paper. The main drawback of RO methods is the inefficiency to include the effects of frequency. However, the results generated with RO methods show high consistency with PE results as in Figure 3 as suggested in [38].
In [43], the earth is assumed as flat for the ray-tracing simulations. However, the curvature of the earth becomes important at b-LoS distances. Therefore, we utilize the earth radius transformation and the Snell's law to find the Eikonal equation for surface ducts by assuming the atmosphere has infinitesimal stratified layers. In this way, ray trajectories can be estimated with the Eikonel equation which is represented as

$$
\frac{1}{n(z)} \frac{d n}{d z}-\frac{1}{R_{0}}=\frac{d^{2} z}{d x^{2}},
$$

where $R_{0}$ is the radius of the earth which is used as $6370 \mathrm{~km}$, and the derivation of (6) can be found in Appendix A. $n(z)$ shows very slight variations with the height which is in the order of $10^{-3}$ [44]. Therefore, (6) is simplified as

$$
\frac{1}{n(0)} \frac{d n}{d z}-\frac{1}{R_{0}}=\frac{d^{2} z}{d x^{2}},
$$

where $n(0)$ is used as 1.00035 [44].

Since surface ducts have a bi-linear refractivity model as in Figure 2, (6) can be solved as described in Appendix B and ray trajectories are calculated as

$$
z=\frac{1}{2}\left(\frac{1}{n(0)} \frac{d n}{d z}-\frac{1}{R_{0}}\right) x^{2}+\theta x+h_{t},
$$

where $\theta$ is the angle of the ray with respect to the ground at the transmitter. (8) can be solved step by step for each ray to calculate the trajectory of the trapped paths.

Figure 3(b) shows the ray trajectories for a $40 \mathrm{~m}$ surface duct $(\Delta M=20 \mathrm{M}$-unit), and Figure 3(a) presents path-loss results for the same ducting conditions by using PETOOL with the following channel parameters: $27 \mathrm{~m}$ transmitter, horizontal polarization, and $10 \mathrm{GHz}$. As noticed, regions with high ray densities are associated with low path-loss values. In addition, shadowed regions are located at the similar locations at both figures. Therefore, PE methods and RO methods give consistent results.

2) Trapping Beam-width: According to the channel geometry and atmospheric conditions, only rays that have certain angles can be trapped by atmospheric ducts. Rays outside of this certain range cannot be trapped and propagate through the atmosphere. Energy of these rays are wasted in b-LoS systems. 
To increase the efficiency, we develop an analytical model to estimate the effective trapping beam-width at the transmitter side for the first time in the literature.

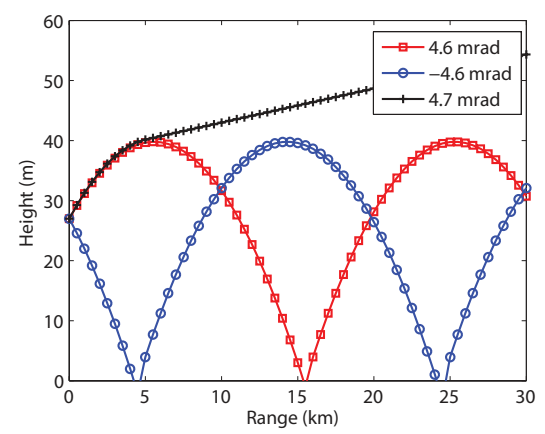

Fig. 4. Ray trajectories with the ray-tracing method.

By utilizing the proposed ray trajectory formula (8), we derive the span of effective trapped beamwidths which depends on the change in the refractive index, transmitter height and duct height. The derivation of maximum and minimum trapping beam-widths $\left(\theta_{\max , \min }^{T}\right)$ can be found in Appendix $\mathrm{C}$, and $\theta_{\max , \min }^{T}$ are found as

$$
\theta_{\text {max } \min }^{T}= \pm \sqrt{2\left(\frac{1}{n(0)} \frac{d n}{d z}-\frac{1}{R_{0}}\right)\left(h_{t}-\delta\right)} .
$$

In trapping layer, refractive index decreases with height, and this implies that $\frac{d n}{d z}<0$. In addition, our model assumes that transmitter is located within surface duct $\left(h_{t}<\delta\right)$. Therefore, the term inside the square-root in (9) is positive, and it has two real roots.

According to (9), a $40 \mathrm{~m}$ surface duct with $\Delta M=20$ has $\theta_{\text {max }, \text { min }}^{T}= \pm 4.64 \mathrm{mrad}= \pm 0.266^{\circ}$ where the transmitter height is $h_{t}=27 \mathrm{~m}$. We also perform RO simulations based on (8) to verify our analytical trapping beam-width formula. Figure 4 shows the ray trajectories for the angles that are on the boundaries. As noticed, analytically calculated trapping beam-width is consistent with the simulations. As a result, adaptive antenna techniques can be designed for ducting channel systems to maximize the trapped power with this equation because signal transmitted out of this beam-width region cannot be trapped by the surface ducts. In addition, for symmetrical ducting channels, where $h_{t}=h_{r}$ and refractivity profile is range independent, the calculated trapping beamwidths form an upper and lower bound for the AOA in the ducting channel.

\section{LARGE-SCAle Path-Loss Model for Surface DUCTS}

Ducting channel wave propagation depends on many factors: transmitter height, receiver height, duct height, duct strength, carrier frequency, polarization and surface conditions. PE based methods are capable of taking all of these parameters as input. Thus, we utilize PETOOL to solve PE to estimate path-loss in surface ducts for given channel parameters. We aim to develop a path-loss model as a function the channel parameters: transmitter height, receiver height, duct height, duct strength and carrier frequency for the first time in the literature. Our model will be independent of polarization because the effects of polarization is not noticeable in the large-scale, and we use only horizontal polarization for both of the antennas. In addition, surface is assumed as smooth sea surface.

We aim to model path-loss in ducting channel for b-LoS distances where Line-of-Sight (LoS) transmission is impossible in the standard atmospheric conditions. For b-LoS ducting channel, the large-scale path-loss equation is given as [45]

$$
P L=A+10 \gamma \log d / d_{0}+X_{s},
$$

where $\gamma$ is the path-loss exponent, $d_{0}$ is the b-LoS range, $A$ is the path-loss at $d_{0}$ and $X_{s}$ is the shadow fading. The bLoS range is given by the well-known maximum LoS range equation for the standard refractivity conditions as

$$
d_{0}=4.12 \sqrt{h_{t}}+4.12 \sqrt{h_{r}},
$$

where $h_{t}$ and $h_{r}$ are the transmitter and receiver heights, respectively.

In this section, we develop a statistical large-scale pathloss model based on PE simulations performed with PETOOL. We perform well-known regression methods to the results of PETOOL. Similar approach can be found in [46]. In addition, the distribution of shadow fading and the coefficients of the channel parameters are determined by using the multivariate linear regression method to (10). To this end, we perform PE simulations for the path-loss of the link for different channel conditions. Figure 5(a) contains the path-loss results generated with PETOOL, the free space path-loss and the fitted line to the PETOOL results for (10) with simulation parameters: 500 $\mathrm{km}$ range, $40 \mathrm{~m}$ surface duct, $27 \mathrm{~m}$ transmitter and receiver, 10 $\mathrm{GHz}$ carrier frequency, $20 \mathrm{M}$-unit duct strength and horizontal polarization. For the regression line fitting for (10), we utilize the least-square fitting. Deviations from the fitting line is treated as shadow fading, and the distribution of shadow fading can be seen in Figure 5(b). Shadowed regions can be clearly seen in Figure 3.

Since path-loss enhancements with atmospheric ducts are most effective when both of the antennas are in ducting layer [28], we assume that both transmitter and receiver are located within surface duct. To develop the large-scale pathloss model, we perform extensive simulations by varying the channel parameters: carrier frequency $(f=5-15 \mathrm{GHz})$, duct height $(\delta=10-60 \mathrm{~m})$, duct strength rate $(\Delta M / \delta=0.1-0.3$ M-unit/m), transmitter height $\left(h_{t}=0-\delta \mathrm{m}\right)$ and receiver height $\left(h_{r}=0-\delta \mathrm{m}\right)$. The remaining of the section includes the derivation of the coefficients and the statistical distribution of the large-scale path-loss model parameters: $A, \gamma$ and $X_{s}$.

\section{A. A Parameter}

After performing a number of simulations by using PETOOL, we try to find the statistical distribution of each parameter. Since ducting channel that we are trying to model is symmetric, the effect of transmitter and receiver antenna heights can be modeled with the difference between them $\Delta h=h_{t}-h_{r}$. We use multivariate regression analysis in 


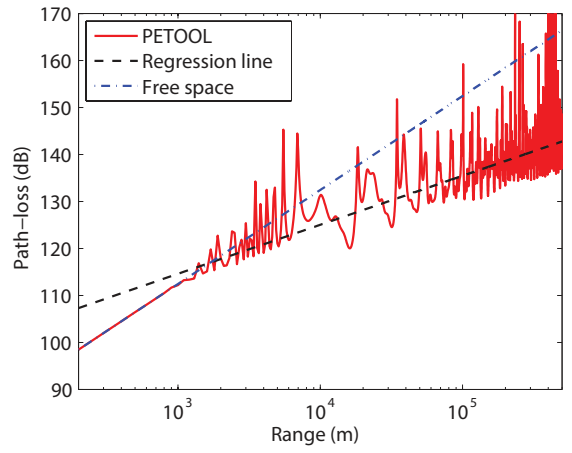

(a)

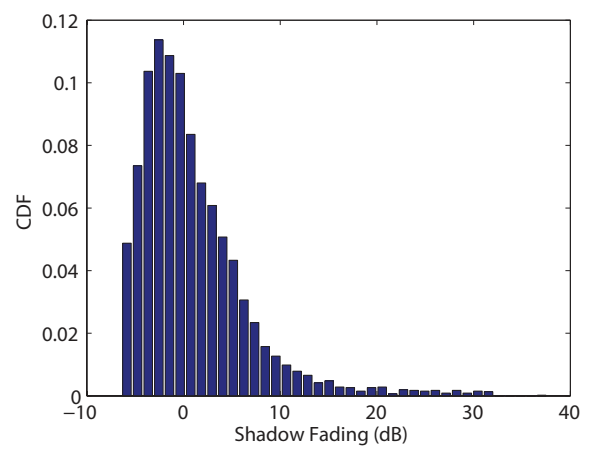

(b)

Fig. 5. The path loss results for (a) the regression method and, (b) the distribution of the shadow fading.

the least-square error sense to find the coefficients of each channel parameter. $A$ parameter is modeled as

$$
\left[\begin{array}{ccccc}
1 & \vdots & \vdots & \vdots & \vdots \\
\vdots & \Delta h_{i} & \delta_{i} & f_{i} & \Delta m \\
1 & \vdots & \vdots & \vdots & \vdots
\end{array}\right]\left[\begin{array}{c}
\alpha_{A} \\
\beta_{A} \\
\kappa_{A} \\
\xi_{A} \\
\varrho_{A}
\end{array}\right]=\left[\begin{array}{c}
\vdots \\
A_{i} \\
\vdots
\end{array}\right],
$$

where $\Delta h$ is the height difference between the transmitter and the receiver, $\delta$ is the duct height, $f$ is the carrier frequency, $\Delta m$ is the change in the modified refractivity index per $\mathrm{m}$ and $A_{i}$ is the resulting value for each simulation case. By performing multivariate regression analysis in the least-square error sense, the resulting coefficients are found as $\alpha_{A}=123.34 \mathrm{~dB}, \beta_{A}=$ $0.015 \mathrm{~dB} / \mathrm{m}, \kappa_{A}=0.13 \mathrm{~dB} / \mathrm{m}, \xi_{A}=8.66 \times 10^{-4} \mathrm{~dB} / \mathrm{MHz}$ and $\varrho_{A}=-12.83 \mathrm{~dB} \times \mathrm{m}$. The variation (error) of $A$ from the regression model is found as

$$
\Delta A=\left[\begin{array}{c}
\vdots \\
A_{i} \\
\vdots
\end{array}\right]-\left[\begin{array}{ccccc}
1 & \vdots & \vdots & \vdots & \vdots \\
\vdots & \Delta h_{i} & \delta_{i} & f_{i} & \Delta n \\
1 & \vdots & \vdots & \vdots & \vdots
\end{array}\right]\left[\begin{array}{c}
\alpha_{A} \\
\beta_{A} \\
\kappa_{A} \\
\xi_{A} \\
\varrho_{A}
\end{array}\right] .
$$

Figure 6(a) represents the deviation of $A$ from our model and $\triangle A$ clearly shows a Gaussian distribution with the zero mean and standard deviation $\sigma_{\Delta A}=2.39 \mathrm{~dB}$. Therefore, $A$ can be represented as

$$
A \mathrm{~dB}=\alpha_{A}+\beta_{A} \Delta h+\kappa_{A} \delta+\xi_{A} f+\varrho_{A} \Delta m+\sigma_{\Delta A} x,
$$

where $x$ is zero mean unit variance Gaussian random variable.

\section{B. $\gamma$ Parameter}

By the same method, $\gamma$ parameter is modeled as

$$
\left[\begin{array}{ccccc}
1 & \vdots & \vdots & \vdots & \vdots \\
\vdots & \Delta h_{i} & \delta_{i} & f_{i}^{2} & \Delta m \\
1 & \vdots & \vdots & \vdots & \vdots
\end{array}\right]\left[\begin{array}{c}
\alpha_{\gamma} \\
\beta_{\gamma} \\
\kappa_{\gamma} \\
\xi_{\gamma} \\
\varrho_{\gamma}
\end{array}\right]=\left[\begin{array}{c}
\vdots \\
\gamma_{i} \\
\vdots
\end{array}\right] .
$$

According to the regression results, the resulting coefficients is found as $\alpha_{\gamma}=1.3, \beta_{\gamma}=2 \times 10^{-3} 1 / \mathrm{m}, \kappa_{\gamma}=-2.9 \times$ $10^{-3} 1 / \mathrm{m}, \xi_{\gamma}=-3.47 \times 10^{-10} 1 / \mathrm{MHz}, \varrho_{\gamma}=-0.58 \mathrm{~m}$. The coefficients $\beta_{\gamma}$ and $\kappa_{\gamma}$ result in too low values when they are multiplied with their parameters. Therefore, the effects of these factors can be neglected at the regression method. In (15), the frequency term is squared in order to make the error term as close as possible to the Gaussian distribution and as can be seen in Figure 6(b), $\Delta \gamma$ has a Gaussian distribution with zero mean and $\sigma_{\Delta \gamma}=0.066$. In addition, the negative $\varrho_{\gamma}$ indicates that increasing duct strength reduces the path-loss exponent. Therefore, $\gamma$ can be modeled as

$$
\gamma=\alpha_{\gamma}+\xi_{\gamma} f^{2}+\varrho_{\gamma} \Delta m+\sigma_{\Delta \gamma} y
$$

where $y$ is zero mean unit variance Gaussian random variable.

\section{Shadowing Fading}

As shown in Figure 5(a), there is a significant shadow fading in the link, and also the distribution of the shadow fading is shown in Figure 5(b). The best fitted distribution for the shadow fading in the channel can be found with the Kullback-Leibler divergence (KLD) [47]. Let $P$ and $Q$ be two distributions and the divergence of $Q$ from $P$ can be calculated as

$$
D_{K L}(P \| Q)=\sum_{i} \ln \frac{P(i)}{Q(i)} P(i) .
$$

$D_{K L}(P \| Q)$ takes lower values when the two distribution get closer, and it becomes 0 for the identical distributions.

We generate various distributions with MATLAB and use (17) to determine how close the generated distributions to the shadow fading are. The simulation results for the possible distributions can be found in Table I. According to our results, the Weibull distribution best matches with the shadow fading for surface ducts. Therefore, the shadow fading in the channel is modeled with log-Weibull distribution which is also known as Gumbel distribution.

The PDF of the Weibull distribution can be represented as

$$
f(x ; \lambda, k)= \begin{cases}\frac{k}{\lambda}\left(\frac{x}{\lambda}\right)^{k-1} e^{-(x / \lambda)^{k}}, & x \geq 0 \\ 0, & x<0\end{cases}
$$

where $k>0$ is the shape parameter and $\lambda>0$ is the scale parameter. We perform the multivariate regression analysis to find the distribution of $k$ and $\lambda$ as well. 


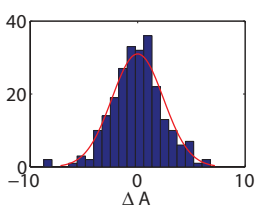

(a) Distribution of $\Delta A$.

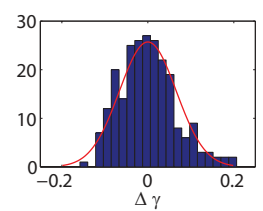

(b) Distribution of $\Delta \gamma$.

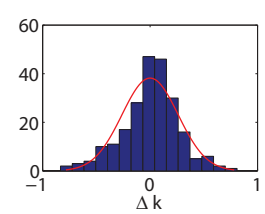

(c) Distribution of $\Delta k$.

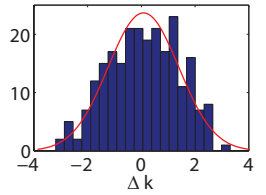

(d) Distribution of $\Delta \lambda$.

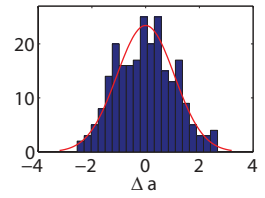

(e) Distribution of $\Delta a$.

Fig. 6. The distributions for the deviations from our multivariate regression model.

TABLE I

THE KLD RESULTS.

\begin{tabular}{|l|c|}
\hline Type of Distribution & KLD \\
\hline Normal distribution & 1.3436 \\
\hline Rayleigh distribution & 0.1954 \\
\hline Weibull distribution & $\mathbf{0 . 0 2 0 9}$ \\
\hline Gamma distribution & 0.0283 \\
\hline Generalized extreme value distribution & 0.0887 \\
\hline Nakagami distribution & 0.0574 \\
\hline
\end{tabular}

We use the same methodology as in $A$ and $\gamma$ parameters. Again, the parameters with slight effects are neglected and only the final results are presented for the remaining multivariate fitting results. With the multivariate linear regression analysis, the $k$ parameter can be given as

$$
k=\alpha_{k}+\xi_{k} f^{3}+\varrho_{k} \Delta m+\sigma_{\Delta k} z,
$$

where $z$ is the zero mean and unit variance Gaussian random variable. $\alpha_{k}=0.1792, \xi_{k}=3.36 \times 10^{-14} 1 / \mathrm{MHz}$ and $\varrho_{k}=$ $1.85 \mathrm{~m}$. The deviation of $k$ from our model can be found in Figure 6(c), and this deviations can be modeled with Gaussian distribution with zero mean $\sigma_{\Delta k}=0.26$.

The $\lambda$ parameter can be modeled as

$$
\lambda=\alpha_{\lambda}+\beta_{\lambda} \delta+\xi_{\lambda} f^{3}+\varrho_{\lambda} \Delta m+\sigma_{\Delta \lambda} q,
$$

where $q$ is the zero mean and unit variance Gaussian random variable. $\alpha_{\lambda}=-1.94, \kappa_{\lambda}=0.1761 / \mathrm{m}, \xi_{\lambda}=1.09 \times 10^{-12}$ 1/MHz. Figure 6(d) shows the deviations of the $\lambda$ from our model, and it has Gaussian distribution with zero mean and $\sigma_{\Delta \lambda}=1.45$.

As noticed in Figure 5(b), the shadow fading values can be negative. However, since the Weibull distribution takes only positive values, we first shift the shadow fading to the right by $\Delta a$ and perform the distribution fitting. To this end, $\Delta a$ can be represented as

$$
\Delta a \mathrm{~dB}=\alpha_{a}+\beta_{a} \delta+\kappa_{a} f+\varrho \Delta m+\sigma_{\Delta a} w,
$$

where $w$ is the zero mean and unit variance Gaussian random variable. The resulting values of the $\alpha_{a}=3.27 \mathrm{~dB}, \kappa_{a}=$ $-0.141 / \mathrm{m}, \xi_{a}=3.2 \times 10^{-4} 1 / \mathrm{MHz}$, and $\sigma_{\Delta a}=1.07 \mathrm{~dB}$ as can be seen in Figure 6(e).

\section{The large-scale Path-Loss Model}

By combining (10), (14), (16), and (21), the statistical largescale path-loss equation is found as

$$
\begin{aligned}
P L= & A+10 \gamma_{\Delta h} \log d / d_{0}+X_{s} \\
P L= & \alpha_{A}+\beta_{A} \Delta h+\kappa_{A} \delta+\xi_{A} f+\varrho_{A} \Delta m+\sigma_{\Delta A} x+ \\
& +10\left(\alpha_{\gamma}+\xi_{\gamma} f^{2}+\varrho_{\gamma} \Delta m+\sigma_{\Delta \gamma} y\right) \log d / d_{0}+ \\
& W+\alpha_{a}+\beta_{a} \delta+\kappa_{a} f+\varrho \Delta m+\sigma_{\Delta a} z
\end{aligned}
$$

where $x, y$ and $z$ are the zero mean unit variance independent normal random variables and $W$ has the Weibull distribution with $k$ in (19) and $\lambda$ in (20) .

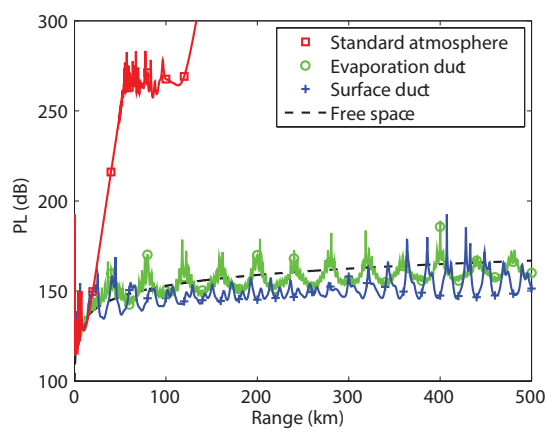

Fig. 7. Path-loss vs. range for standard atmosphere, free space, and atmospheric ducts.

\section{Simulation Results}

In this section, we present the simulation results with the PE methods and RO methods. In addition, the results of the large-scale path-loss model is compared with the PE results. We present link budget calculations for surface ducts to predict the communication ranges for given channel parameters.

\section{A. Simulation Results with PEM}

We utilize PETOOL to perform the PE simulations as described in Section IV-A. Figure 7 presents the path-loss vs. range for different atmospheric ducts, the free space and the standard atmosphere at $10.5 \mathrm{GHz}, 5 \mathrm{~m}$ transmitter and receiver, and horizontal polarizations. As noticed, the curves for 40 $\mathrm{m}$ evaporation duct and $40 \mathrm{~m}$ surface duct $(\Delta M=10 \mathrm{M}$ unit) outperform the standard atmosphere in terms of path-loss values. In addition, surface ducts have strong shadow fading compared to the evaporation ducts.

Figure 8(a) shows the path-loss for a $40 \mathrm{~m}$ surface duct (20 M-units) as a function of the carrier frequency and receiver height. As noticed, the lower frequencies $3-5 \mathrm{GHz}$ have significantly higher trapping capabilities compared to the evaporation ducts [3], [16]. This is one the main difference between surface ducts and evaporation ducts in terms of channel modeling because evaporation ducts provide lowest path-loss values near $10 \mathrm{GHz}$. Surface ducts show higher trapping capabilities over evaporation ducts due to the sharp refractivity curve of surface ducts as described in Section III.

Figure $8(\mathrm{~b})$ presents the path-loss values as a function of receiver height. As noticed, the path-loss values for the surface 


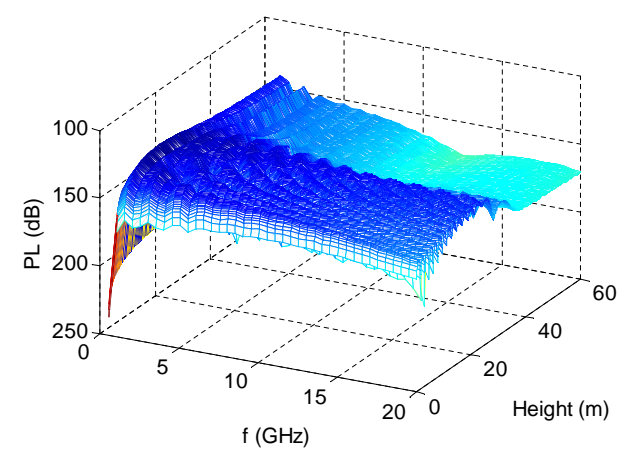

(a) Path-loss vs. receiver height and frequency at $100 \mathrm{~km}$ range.

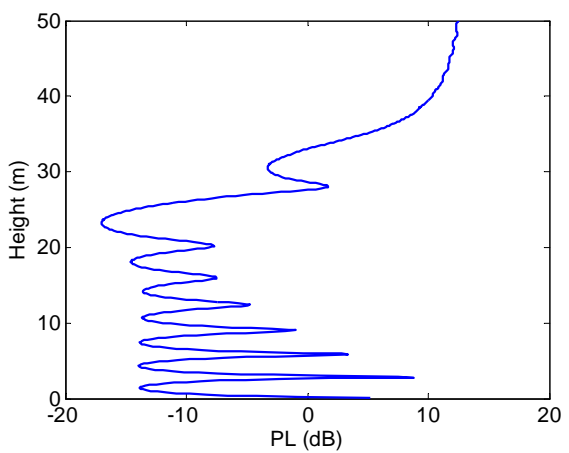

(b) Receiver height vs. path-loss at $100 \mathrm{~km}$ range.

Fig. 8. Path-loss results for $40 \mathrm{~m}$ surface duct, $27 \mathrm{~m}$ transmitter height with PETOOL.

duct is significantly lower compared to the free space pathloss up to $18 \mathrm{~dB}$. However, the power levels have fading with respect to the vertical height. This condition is caused by the constructive/destructive interference of the delayed signals. The delay spread of the channel should be examined in order to determine the fading behavior of the channel. Therefore, the next subsection presents the RO results to analyze the delay spread of the ducting channel.

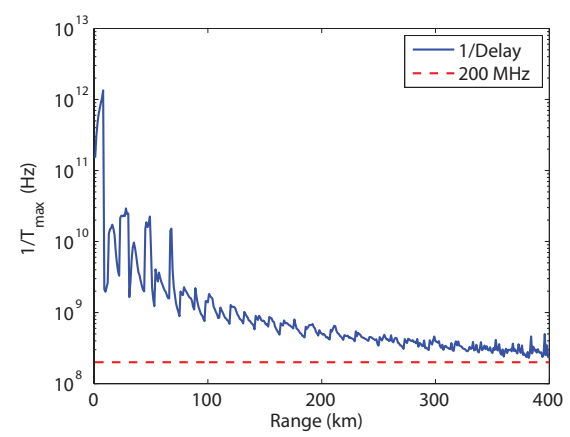

Fig. 9. $1 / T_{\max }$ spread for a $40 \mathrm{~m}$ surface duct.

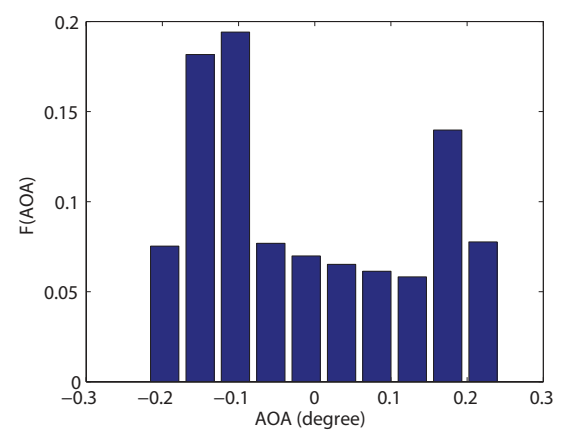

Fig. 10. Histogram of AOA for a $40 \mathrm{~m}$ surface duct.

\section{B. Simulation Results with ROM}

By using the calculated ray trajectories as in Figure 3(b), delay spreads and AOAs of the received rays can be calculated.
Figure 9 presents $1 / T_{\max }$ in $\mathrm{Hz}$ vs. the range for $27 \mathrm{~m}$ transmitter and receiver with a $40 \mathrm{~m}$ surface duct $(\Delta M=20$ M-units) where $T_{\max }$ is the maximum delay spread. As noticed from the figure, $1 / T_{\max }$ value is higher than $200 \mathrm{MHz}$ line up to $400 \mathrm{~km}$. Since the channel bandwidth should be much lower than $1 / T_{\max }$ for a channel to have flat fading, the ducting channel can be assumed as a flat fading channel up to $400 \mathrm{~km}$ with $20 \mathrm{MHz}$ bandwidth.

Figure 10 presents the AOA of the ducting channel for $27 \mathrm{~m}$ transmitter and receiver with a $40 \mathrm{~m}$ surface duct $(\Delta M=20$ M-units). As noticed, the AOA results are bounded by the analytical trapping beam-width value of $0.266^{\circ}$ calculated previously in Section IV-B2.

\section{Large-scale Path-loss Model}

We compare the results of our large-scale path-loss model with PETOOL for verification. The following channel parameters are utilized in the simulations: $f=10 \mathrm{GHz}, h_{t}=h_{r}=$ $20, \delta=40 \mathrm{~m}, \Delta M=20$ M-unit, horizontal polarization. Figure 11 includes the comparison of the results in the upper figures. As noticed, our model can predict the level of the path loss for each range. The lower figures show the distribution of the deviations from our proposed model which can be modeled as the small-scale fading. As noticed, the normalized deviations can be modeled with Rayleigh distribution, and the distribution becomes closer to the Rayleigh distribution with the increasing range.

\section{Link Budget Calculations}

The link budget calculations are presented in this section, and the following parameters are utilized in the b-LoS channel with surface ducts:

- Transmit power $\left(P_{t}\right)=30 \mathrm{dBm}(1 \mathrm{~W})$

- Antenna gains $\left(G_{t, r}\right)=30 \mathrm{~dB}$

- Cable losses $\left(L_{q}\right)=10 \mathrm{~dB}$

Received power is given by

$$
P_{r d B m}=P_{t d B m}+G_{t d B}-P L_{d B}+G_{r d B}-L_{q_{d B}} .
$$

The modern modems requires a minimum received power of $-80 \mathrm{dBm}$ to operate at Mbps data rates [48]. Thus, the 


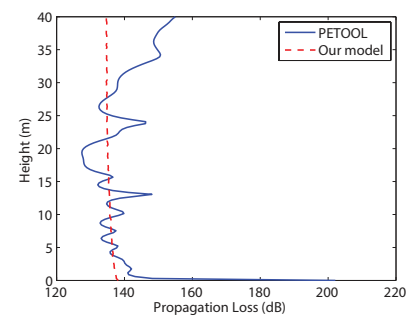

(a) Comparision at $50 \mathrm{~km}$.

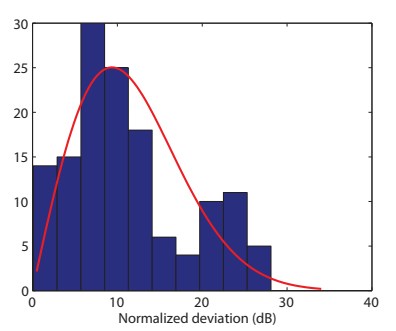

(d) Histogram of deviation at $50 \mathrm{~km}$.

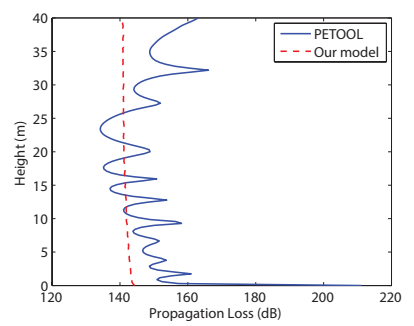

(b) Comparision at $250 \mathrm{~km}$.

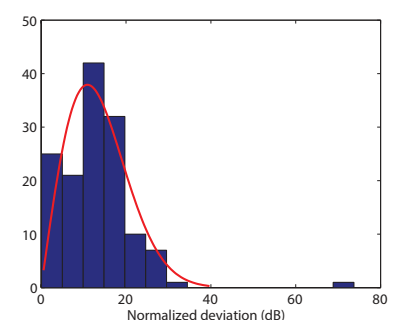

(e) Histogram of deviation at $250 \mathrm{~km}$.

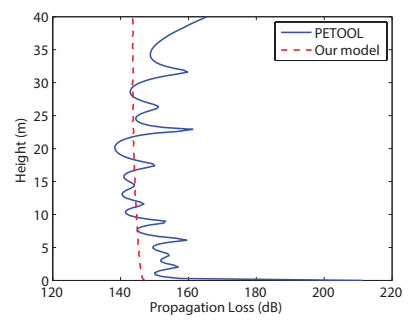

(c) Comparision at $500 \mathrm{~km}$.

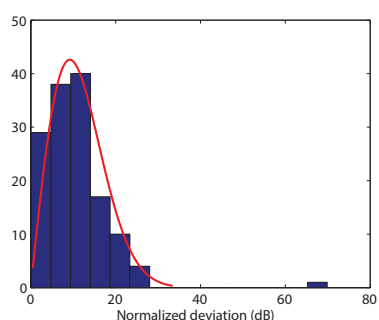

(f) Histogram of deviation at $500 \mathrm{~km}$.

Fig. 11. The comparison of our large-scale path-loss model and PETOOL results.

maximum $P L$ that can provide the communication link is found as $160 \mathrm{~dB}$ (PL threshold) by (23). Figure 12 presents the path-loss curves for different frequencies for $90 \%$ not exceeded of the time and $P L$ threshold for a $40 \mathrm{~m}$ surface duct $(\Delta M=10), 27 \mathrm{~m}$ transmitter and receiver. As noticed, 10 $\mathrm{GHz}$ can provide communication links up to $1000 \mathrm{~km}$. Lower frequencies can provide higher ranges. For high duct heights $(>20 \mathrm{~m})$, lower frequencies have better trapping capabilities as also experimentally presented in [49]. Since duct height of surface ducts are generally between $20-60 \mathrm{~m}$ as in [11], [12], lower frequencies will show better trapping capabilities for the surface ducts. However, duct height for evaporation ducts is mostly smaller than $20 \mathrm{~m}$. Thus, trapping capability of evaporation ducts significantly differs from the surface ducts as presented in [2], [16].

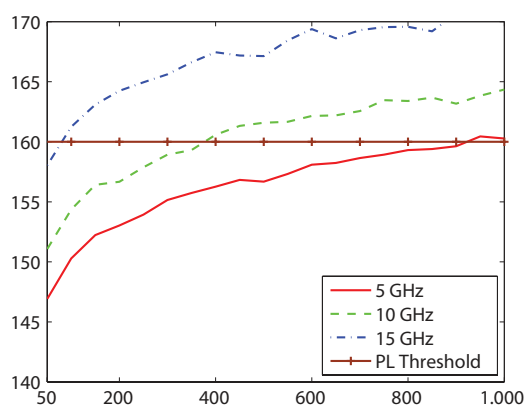

Fig. 12. Link budget analysis for surface ducts.

\section{CONClusions And Future Work}

In this work, we develop a large-scale path-loss model for the surface ducts and analyze the delay spreads and AOA of the ducting channel. According to our results, the ducting channel can be used as communication medium up to $1000 \mathrm{~km}$, and the small-scale fading in the channel shows Rayleigh fading.
In addition, our RO methods can be utilized to estimate the fading behavior of the channel. Upper and lower bounds for the AOA of the channel can be estimated with the effective trapping beam-width equation.

\section{APPENDIX A}

\section{DERIVATION OF THE EIKONAL EQUATION}

To derive (6), the earth radius transformation is utilized as shown in Figure 13 [39]. Therefore, the well-known Snell's equation for the stratified atmosphere is given as

$$
R_{0} n(z) \cos (\theta)=\left(R_{0}+\Delta R\right) n(z+\Delta z) \cos (\theta-\Delta \theta) .
$$

Since the index of refraction is modeled with a bi- linear curve for the surface ducts, (24) is represented as

$$
R_{0} n(z) \cos (\theta)=\left(R_{0}+\Delta R\right)\left(n(z)-\frac{d n}{d z} \Delta z\right) \cos (\theta-\Delta \theta) .
$$

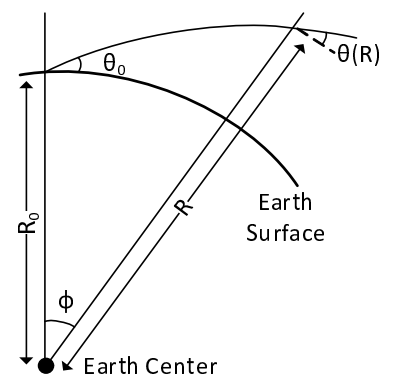

Fig. 13. Ray trajectory for refractive path.

We know that $\Delta z$ and $\Delta \theta$ will be small because the step sizes in the simulations will be low. Therefore, their squares and multiplications will give zero $\Delta z^{2}=\Delta \theta^{2}=\Delta z \Delta \theta=0$. For small $\Delta \theta, \cos (\Delta \theta)=1$ and $\sin (\Delta \theta)=\Delta \theta$. By knowing 
these conditions, (25) is simplified as

$$
\begin{aligned}
R_{0} n(z) \cos (\theta) & =R_{0} n(z) \cos (\theta)-R_{0} \frac{d n}{d z} \Delta z \cos (\theta)+ \\
& +\Delta z n(z) \cos (\theta)+R_{0} n(z) \Delta \theta \sin (\theta), \\
& \frac{1}{n(z)} \frac{d n}{d z}=\frac{1}{R_{0}}+\frac{\Delta \theta}{\Delta z} \tan (\theta),
\end{aligned}
$$

where $\frac{\Delta \theta}{\Delta z} \tan (\theta)=\frac{\Delta \theta}{\Delta z} \frac{\Delta z}{\Delta x}=\frac{\Delta \theta}{\Delta x}$ [43]. As $\Delta x$ goes to 0 , $\frac{\Delta \theta}{\Delta x}=\frac{d \theta^{z}}{d x}$. Since $\theta=\frac{d z}{d x}$, the Eikonal equation for the Earth radius transformation can be represented as

$$
\frac{1}{n(z)} \frac{d n}{d z}-\frac{1}{R_{0}}=\frac{d^{2} z}{d x^{2}} .
$$

\section{APPENDIX B}

\section{DERIVATION OF RAY TRAJECTORY EQUATION}

The ray trajectory equation (7) can be solved as

$$
\begin{aligned}
\frac{d^{2} z}{d x^{2}} & =\frac{1}{n(0)} \frac{d n}{d z}-\frac{1}{R_{0}} \\
z^{\prime \prime} & =\frac{1}{n(0)} \frac{d n}{d z}-\frac{1}{R_{0}} \\
z^{\prime} & =\left(\frac{1}{n(0)} \frac{d n}{d z}-\frac{1}{R_{0}}\right) x+\theta, \\
z & =\frac{1}{2}\left(\frac{1}{n(0)} \frac{d n}{d z}-\frac{1}{R_{0}}\right) x^{2}+\theta x+h_{t} .
\end{aligned}
$$

\section{APPENDIX C}

\section{DERIVATION OF TRAPPING BEAM-WIDTH DERIVATIONS}

In the trapping beam-width calculations, it is assumed that antennas has 0 elevation angle. The procedure is designed to find the maximum beam-width that can be trapped by the surface ducts with respect to duct height $(\delta)$ and transmitter antenna height $\left(h_{t}\right)$ as shown in Figure 14.

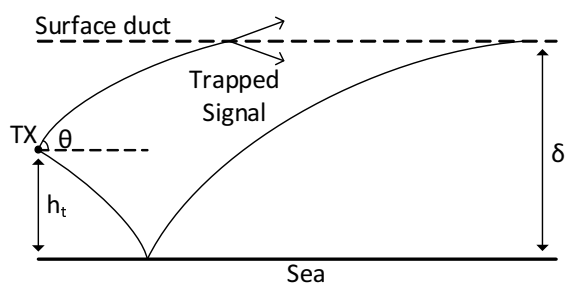

Fig. 14. Trajectory of a trapped ray.

Starting form (8), lets denote $K=\frac{1}{2}\left(\frac{1}{n(0)} \frac{d n}{d z}-\frac{1}{R_{0}}\right)$ and we can write the following equations

$$
\begin{aligned}
z & =K x^{2}+\theta x+h_{t} \\
z+\Delta z & =K(x+\Delta x)^{2}+\theta(x+\Delta x)+h_{t} .
\end{aligned}
$$

If we subtract the above equations, the result is given as

$$
\begin{aligned}
\Delta z & =\Delta x^{2} K+2 x \Delta x K+\theta \Delta x, \\
\frac{\Delta z}{\Delta x} & =\Delta x K+2 x K+\theta .
\end{aligned}
$$

At the boundary layer, the trapped ray will have zero $\Delta z$ for very small step sizes because the trapped signal will first become parallel with the surface and then propagate downward. In addition, for small step sizes, $K \Delta x$ term will be nearly zero. Therefore, (36) is simplified as

$$
\begin{aligned}
-2 x K & =\theta^{T}, \\
x & =-\left(\theta^{T} / 2 K\right) .
\end{aligned}
$$

Therefore, at the duct height (boundary $z=\delta$ ), (33) is represented as

$$
z=\delta=K x^{2}+\theta^{T} x+h_{t}
$$

By combining (38) and (39), the maximum and minimum effective trapping beam-widths are found as

$$
\begin{aligned}
\delta & =(1 / 4 K) \theta^{T^{2}}-(1 / 2 K) \theta^{T^{2}}+h_{t}, \\
\delta-h_{t} & =-(1 / 4 K) \theta^{T^{2}}, \\
\theta^{T^{2}} & =4 K\left(h_{t}-\delta\right), \\
\theta_{\max , \min }^{T} & = \pm \sqrt{4 K\left(h_{t}-\delta\right)}, \\
\theta_{\max , \min }^{T} & = \pm \sqrt{2\left(\frac{1}{n(0)} \frac{d n}{d z}-\frac{1}{R_{0}}\right)\left(h_{t}-\delta\right) .}
\end{aligned}
$$

Since the transmitter located within duct both $\frac{d n}{d z}$ and $h_{t}-\delta$ terms are negative, so this equation will have two real roots.

\section{REFERENCES}

[1] H. V. Hitney, J. H. Richter, R. A. Pappert, K. D. Anderson, G. B. Jr Baumgartner, "Tropospheric radio propagation assessment," Proceedings of the IEEE, vol. 73, no. 2, pp. 265-283, Feb. 1985.

[2] G. S. Woods, A. Ruxton, C. Huddlestone-Holmes, G. Gigan, "HighCapacity, Long-Range, Over Ocean Microwave Link Using the Evaporation Duct,' IEEE Journal of Oceanic Engineering, vol. 34, no. 3, pp. 323-330, July 2009.

[3] E. Dinc, O. B. Akan, "Beyond-Line-of-Sight Communications with Ducting Layer," IEEE Communications Magazine, vol. 52, no. 10, pp. 37-43, October 2014.

[4] I. Sirkova, "Brief review on PE method application to propagation channel modeling in sea environment" Central European Journal of Engineering, vol. 2, issue 1, pp. 19-38, 2012.

[5] J. H. Richter, "Sensing of radio refractivity and aerosol extinction," International Geoscience and Remote Sensing Symposium, vol. 1, pp. 381-385, Pasadena, CA, Aug. 8-12, 1994.

[6] A. Willitsford, C. R. Philbrick, "Lidar description of the evaporative duct in ocean environments," Proceedings of SPIE, vol. 5885, Bellingham, WA, Sep. 2005.

[7] R. M. Hodur, "The naval research laboratorys coupled ocean/atmos-phere mesoscale prediction system (COAMPS), Monthly Weather Rev., vol. 125, no. 7, pp. 14141430, 1996.

[8] A. R. Lowry, C. Rocken, S. V. Sokolovskiy, and K. D. Anderson, "Vertical profiling of atmospheric refractivity from ground-based GPS, Radio Sci., vol. 37, no. 3, pp. 10411059, 2002.

[9] L. T. Rogers, C. P. Hattan, and J. K. Stapleton, "Estimating evaporation duct heights from radar sea echo, Radio Sci., vol. 35, no. 4, pp. 955966, 2000.

[10] P. Gerstoft, L. T. Rogers, J. L. Krolik, and W. S. Hodgkiss, "Inversion for refractivity parameters from radar sea clutter, Radio Sci., vol. 38, no. 3, pp. 122, Apr. 2003.

[11] S. S. Mentes, Z. Kaymaz, "Investigation of Surface Duct Conditions over Istanbul, Turkey," Journal of Applied Meteorology and Climatology, vol. 46, pp 318-338, 2007.

[12] S. M. Babin "Surface Duct Height Distributions for Wallops Island, Virginia, 1985-1994," J. Appl. Meteor., vol. 35, pp. 8693, 1996.

[13] L. M. Brekhovskikh, Waves in layered media, 2nd ed., Academic Press, New York, 1980 
[14] G. B. Baumgartner, H. V. Hitney, R. A. Pappert, "Duct propagation modeling for the Integrated Refractive Effects Prediction System (IREPS)," IEE Proc.-F, vol. 130, pp. 630-642, 1983.

[15] R. H. Hardin and F. D. Tappert, "Application of the split-step Fourier method to the numerical solution of nonlinear and variable coefficient wave equations, SIAM Rev., vol. 15, pp. 423, 1973.

[16] A. Iqbal, V. Jeoti, "Feasibility study of radio links using evaporation duct over sea off Malaysian shores," International Conference on Intelligent and Advanced Systems (ICIAS), pp. 1-5, June 2010.

[17] S. D. Gunashekar, E. M. Warrington, and D. R. Siddle, "Long-term statistics related to evaporation duct propagation of $2 \mathrm{GHz}$ radio waves in the English Channel," Radio Sci., vol. 45, 2010.

[18] Users Manual for Advanced Refractive Effects Prediction System (AREPS) . San Diego, CA: Space and Naval Warfare Systems Center, pp. 17, 2004.

[19] A. E. Barrios, "Considerations in the development of the advanced propagation model (APM) for U.S. Navy applications," Proc. of the Int. Radar Conference, pp. 77-82, 2003.

[20] A. E. Barrios, "Advanced Propagation Model, Proc. Of the 1997 Battlespace Atmospherics Conf., pp. 483-490 Dec 1997.

[21] A. E. Barrios, W. L. Patterson, R. A. Sprague, "Advanced Propagation Model (APM) Version 2.1.04," Computer Software Configuration Item (CSCI) Documents SSC San Diego, technical document 3214, 2007.

[22] A. E. Barrios, K. Anderson, G. Lindem, "Low Altitude Propagation Effects - A Validation Study of the Advanced Propagation Model (APM) for Mobile Radio Applications," IEEE Transactions on Antennas and Propagation, vol. 54, no. 10, pp. 2869-2877, Oct. 2006.

[23] O. Ozgun, G. Apaydin, M. Kuzuoglu, L. Sevgi, "PETOOL: MATLABbased one-way and two-way split-step parabolic equation tool for radiowave propagation over variable terrain", Computer Physics Communications, vol. 182, issue 12, pp. 2638-2654, Dec 2011

[24] K. D. Anderson, "Evaporation Duct Communication: Test Plan", Naval Ocean Systems Center Technical Document 2033, 1991, San Diego, California.

[25] K. D. Anderson, L. T. Rogers, "Evaporation Duct Communication: Tes Plan II", Naval Ocean Systems Center Technical Document 1460, 1991, San Diego, California.

[26] L. T. Rogers, K. D. Anderson, "Evaporation Duct Communication: Measurement Results", Naval Ocean Systems Center Technical Document 1571, 1993, San Diego, California.

[27] K. D. Anderson, "Radar detection of low-altitude targets in a maritime environment," IEEE Transactions on Antennas and Propagation, vol. 43, no. 6, pp. 609-613, Jun. 1995.

[28] G. D. Dockery, "Modeling electromagnetic wave propagation in the troposphere using the parabolic equation," IEEE Transactions on Antennas and Propagation, vol. 36, no. 10, pp. 1464-1470, Oct. 1988.

[29] B. R. Bean, E. J. Dutton, Radio meteorology, Dover Publications, New York, 1966.

[30] R. A. Paulus, "Evaporation duct effects on sea clutter," IEEE Transactions on Antennas and Propagation, vol. 38, no. 11, pp. 1765-1771, Nov 1990.

[31] "ITU-R's Rec. P.453-10: The radio refractive index: its formula and refractivity data," ITU-R, 2012.

[32] V. A. Fock, "Solution of the problem of propagation of electromagnetic waves along the earths surface by method of parabolic equations," J. Phys. USSR, vol. 10, pp. 1335, 1946.

[33] M. Levy, Parabolic Equation Methods for Electromagnetic Wave Propagation. London, U.K., Institution of Electrical Engineers, 2000.

[34] G. D. Dockery, J. R. Kuttler, "An improved-boundary algorithm for Fourier split-step solutions of the parabolic wave equation," IEEE Trans. Ant. and Prop., Vol. 44, No. 12, 1592-1599, 1996.

[35] J. R. Kuttler ,G. D. Dockery , "Theoretical description of the parabolic approximation Fourier split-step method of representing electromagnetic propagation in the troposphere" Radio Sci., vol. 26, pp. 381-393, 1991.

[36] A. E. Barrios, "Parabolic equation modeling in horizontally inhomogeneous environments," IEEE Trans. Ant. and Prop., vol. 40, pp. 791-797, 1992.

[37] R. K. Crane, "Propagation Handbook for Wireles", Electrical engineering and applied signal processing series, CRC press LLC, 2003.

[38] R. Akbarpour, A. R. Webster, "Ray-tracing and parabolic equation methods in the modeling of a tropospheric microwave link," IEEE Transactions on Antennas and Propagation, vol. 53, no. 11, pp. 37853791, Nov. 2005.

[39] S. A. Parl, "Characterization of multipath parameters for line-of-sight microwave propagation," IEEE Transactions on Antennas and Propagation, vol. 31, no. 6, pp. 938-948, Nov. 1983.
[40] L. Pickering, J. K. DeRosa, "Refractive Multipath Model for Line-ofSight Microwave Relay Links," IEEE Transactions on Communications, vol. 27, no. 8, pp. 1174-1182, Aug. 1979.

[41] A. R. Webster, "Raypath parameters in tropospheric multipath propagation," IEEE Transactions on Antennas and Propagation, vol. 30, no. 4, pp. 796-800, Jul. 1982

[42] A. R. Webster, "Angles-of-arrival and delay times on terrestrial line-ofsight microwave links," IEEE Transactions on Antennas and Propagation, vol. 31, no. 1, pp. 12-17, Jan. 1983.

[43] J. A. Caicedo, "Propagation In Evaporation Ducts With Applications To Detecting Sea-Surface Targets Using MIMO Radar Systems," University of Florida, FL, Fall 2011.

[44] C. Yardim, "Statistical estimation and tracking of refractivity from radar clutter," Ph.D. dissertation, Univ. California, San Diego, CA, Mar. 2007.

[45] A. Goldsmith, Wireless Communications, Cambridge University Press, 2005

[46] V. Erceg, L. J. Greenstein, S. Y. Tjandra, S. R. Parkoff, A. Gupta, B. Kulic, A. A. Julius, R. Bianchi, "An empirically based path loss model for wireless channels in suburban environments," IEEE Journal on Selected Areas in Communications, vol. 17, no. 7, pp. 1205-1211, Jul 1999.

[47] S. Boyd, L. Vandenberghe, "Convex Optimization," Cambridge University Press, 2004.

[48] General Dynamics, "Troposcatter modem," Model TM-20 datasheet, Oct. 2008.

[49] H. V. Hitney, R. Vieth, "Statistical assessment of evaporation duct propagation," IEEE Transactions on Antennas and Propagation, vol. 38 , no. 6, pp. 794-799, Jun 1990.

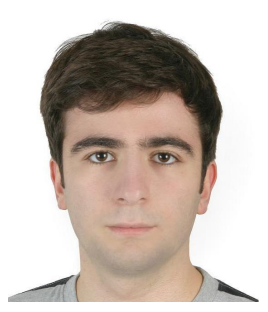

Ergin Dinc [S'12] (edinc@ku.edu.tr) received his B.Sc. degree in Electrical and Electronics Engineering from Bogazici University, Istanbul, Turkey, in 2012. He is currently a research assistant at NextGeneration and Wireless Communications Laboratory (NWCL) and pursuing his Ph.D. degree at the Electrical and Electronics Engineering Department, Koc University, Istanbul, Turkey. His current research interests include communication theory, beyond-Line-of-Sight (b-LoS) communications with troposcatter and atmospheric ducts.

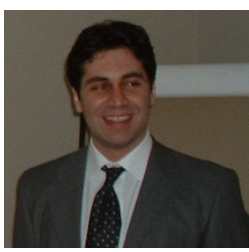

Ozgur B. Akan [M00-SM07] (akan@ku.edu.tr) received his Ph.D. degree in electrical and computer engineering from the Broadband and Wireless Networking Laboratory, School of Electrical and Computer Engineering, Georgia Institute of Technology in 2004. He is currently a full professor with the Department of Electrical and Electronics Engineering, Koc University and the director of the Next-generation and Wireless Communications Laboratory. His current research interests are in wireless communications, nano-scale and molecular communications, and information theory. $\mathrm{He}$ is an Associate Editor of the IEEE Transactions on Communications, the IEEE Transactions on Vehicular Technology, the International Journal of Communication Systems (Wiley), the Nano Communication Networks Journal (Elsevier), and the European Transactions on Technology. 